\title{
Invasive candidosis: contrasting the perceptions of infectious disease physicians and intensive care physicians
}

\author{
Vanessa Schultz ${ }^{[1],[2]}$, Arnaldo Lopes Colombo ${ }^{[3]}$ \\ and Alessandro Comarú Pasqualotto ${ }^{[2],[4]}$
}

[1]. Divisão de Doenças Infecciosas, Santa Casa Complexo Hospitalar, Porto Alegre, RS. [2]. Departamento de Ciências Básicas da Saúde, Universidade Federal de Ciências da Saúde de Porto Alegre, Porto Alegre, RS. [3]. Laboratório de Micologia Molecular, Divisão de Doenças Infecciosas, Universidade Federal de São Paulo, São Paulo, SP. [4]. Laboratório de Biologia Molecular, Santa Casa Complexo Hospitalar, Porto Alegre, RS.

\begin{abstract}
Introduction: We analyze how infectious disease physicians perceive and manage invasive candidosis in Brazil, in comparison to intensive care unit specialists. Methods: A 38-question survey was administered to 56 participants. Questions involved clinicians' perceptions of the epidemiology, diagnosis, treatment and prophylaxis of invasive candidosis. $\mathrm{P}<0.05$ was considered statistically significant. Results: The perception that candidemia not caused by Candida albicans occurs in less than $10 \%$ of patients is more commonly held by intensive care unit specialists $(\mathrm{p}=0.018)$. Infectious disease physicians almost always use antifungal drugs in the treatment of patients with candidemia, and antifungal drugs are not as frequently prescribed by intensive care unit specialists $(\mathrm{p}=0.006)$. Infectious disease physicians often do not use voriconazole when a patient's antifungal treatment has failed with fluconazole, which also differs from the behavior of intensive care unit specialists $(\mathrm{p}=0.019)$. Many intensive care unit specialists use fluconazole to treat candidemia in neutropenic patients previously exposed to fluconazole, in contrast to infectious disease physicians $(\mathrm{p}=0.024)$. Infectious disease physicians prefer echinocandins as a first choice in the treatment of unstable neutropenic patients more frequently than intensive care unit specialists $(p=0.013)$. When candidemia is diagnosed, most infectious disease physicians perform fundoscopy $(\mathrm{p}=0.015)$, whereas intensive care unit specialists usually perform echocardiograms on all patients $(\mathrm{p}=0.054)$. Conclusions: This study reveals a need to better educate physicians in Brazil regarding invasive candidosis. The appropriate management of this disease depends on more drug options being available in our country in addition to global coverage in private and public hospitals, thereby improving health care.
\end{abstract}

Keywords: Candidiasis. Candidemia. Invasive fungal infection. Medical education.

\section{INTRODUCTION}

Invasive fungal diseases have markedly increased in prevalence in recent decades ${ }^{1}$. Candidosis is currently recognized as one of the leading causes of death in critically ill patients. The overall mortality associated with invasive Candida infections is nearly $60 \%$, with an attributable mortality as high as $40 \%^{2}$. In the USA and in many developed countries, Candida spp. have become the fourth leading cause of bloodstream infections (BSIs) ${ }^{3}$. Similar findings have been obtained in studies conducted in Brazil ${ }^{1,2}$. In addition, the costs of modern antifungal therapy have turned candidemia into one of the most expensive infections in clinical practice ${ }^{4}$.

Address to: Dr. Alessandro Comarú Pasqualotto. Laboratório de Biologia Molecular/Hospital Dom Vicente Scherer. Av Independência 155, Heliponto, 90035-075 Porto Alegre, RS, Brasil.

Phone: 5551 9995-1614; Fax: 5551 3213-7491

e-mail: pasqualotto@ufcspa.edu.br

Received 11 March 2013

Accepted 10 July 2013
Understanding how physicians perceive a particular disease is a critical step in establishing priorities for educational programs directed at them. We therefore analyzed how infectious disease (ID) physicians manage invasive candidosis in Brazil, in comparison to intensive care unit (ICU) specialists. Only one similar report was found in the literature in which management choices by both specialties were compared ${ }^{13}$.

\section{METHODS}

In the present study, we sequentially selected ID and ICU physicians working in tertiary care hospitals from three large Brazilian cities (Porto Alegre, Curitiba and São Paulo) who met the following criteria: I) they were board-certified by their respective medical societies; II) they worked in a tertiary care hospital with a minimum of 100 beds and at least one ICU; III) they had at least 3 years of clinical experience in their specialty after finishing medical residency; and IV) they had treated at least one patient with invasive fungal disease in the last year (pneumocystosis not included). Volunteers were mostly recruited during scientific meetings, during the years 
2010-2011, and a single researcher applied the 38-topic survey to all participants. The study included questions related to the clinicians' perceptions on the epidemiology, diagnosis, treatment and prevention of invasive candidosis. Additional questions also addressed the institutions' capability to diagnose invasive Candida infections. This article did not require approval of the ethics committee because the participants were physicians, and any interventions had already been made. All participants freely signed informed consent forms, and their identities were kept secret. Data obtained in the survey were contrasted with the Infectious Diseases Society of America's (IDSA) recommendations for the treatment of candidemia ${ }^{3}$ and also with previously published epidemiological data from the participating centers ${ }^{1,2,5-7}$. Statistical analyses were performed using SPSS version 16.0 for Windows. Categorical variables were compared using chi-squared or Fisher's exact tests, wherever appropriate. $\mathrm{P}<0.05$ was considered statistically significant.

\section{RESULTS}

\section{Participating physicians and institutions}

In total, 56 clinicians from 19 hospitals were interviewed, including 22 ID specialists and 32 ICU physicians. Two physicians were certified in both areas and included in both groups. All participants worked in large tertiary hospitals (median of 700 hospital beds and 33 ICU beds; Table 1). Hospitals were public $(57.1 \%)$, private $(17.9 \%)$, or mixed $(25 \%)$. Most $(92.9 \%)$ institutions were teaching hospitals. The main findings of this study are summarized in Table 2.

\section{Hospitals' capabilities for diagnosing invasive Candida infections}

Automated blood cultures and yeast identification at the species level were available for $96.4 \%$ and $90.9 \%$ of the participants, respectively. The lysis-centrifugation system method was available for $30.3 \%$, whereas 1-3- $\beta$-d-glucan and Candida protein chain reaction (PCR) assays were available for $10.7 \%$ and $36.6 \%$, respectively (with no difference in proportions between medical specialties). Antifungal susceptibility tests were accessible to $49.1 \%$ of the clinicians. The ICU physicians had a higher frequency of compulsory requests for susceptibility tests when candidemia was diagnosed than ID physicians $(62.5 \%$ vs. $44.4 \%$ ), but the difference was not statistically significant $(\mathrm{p}=0.434)$.

\section{Awareness of the epidemiology of invasive Candida infections}

The distribution of Candida species causing candidemia in each medical center was allegedly known by $80 \%$ of participants. However, $69.6 \%$ of the ICU physicians believed that the frequency of non-Candida albicans species was lower than $10 \%$ in their hospitals, in contrast to $31.2 \%$ of ID doctors ( $\mathrm{p}=0.018$ ). The proportion of $C$. glabrata infections causing candidemia was perceived to be lower than $10 \%$ by $90.9 \%$ of participants, with no difference between specialties.

\section{Do physicians always treat patients with positive blood cultures for Candida spp.?}

Whereas $95.8 \%$ of ID specialists affirmed that all patients with candidemia require antifungal treatment, this proportion was only $65.6 \%$ among ICU specialists $(p=0.006)$. For ICU specialists, absence of sepsis was the main reason for potentially not offering antifungal drugs to a patient with candidemia $(63.6 \%)$. Moreover, $45.4 \%$ of the ICU physicians who claimed not to treat their patients with antifungal drugs also reported not asking for routine eye examinations. Fundoscopy was performed systematically when treating a patient with candidemia by only $54.5 \%$ of those physicians surveyed (ID $65.2 \%$, ICU $46.9 \%$; $\mathrm{p}=0.178$ ). The proportions of physicians reporting that eye examination was never requested were $13 \%$ and $43.8 \%$ (ID and ICU, respectively; $\mathrm{p}=0.015$ ).

\section{Primary antifungal treatment choices for candidemia}

Nearly (96.4\%) all participants expressed concerns regarding fluconazole use in patients with invasive Candida infections. However, fluconazole was the preferred antifungal drug for $91.1 \%$ of the interviewed physicians for treating non-neutropenic, stable patients with candidemia. For patients previously exposed to azoles, fluconazole remained the drug of choice for $46.9 \%$ and $12.5 \%$ of the ICU and ID physicians, respectively $(\mathrm{p}=0.024)$. Loading doses of fluconazole were prescribed by only $34.4 \%$ and $58.3 \%$ of the ICU and ID physicians $(\mathrm{p}=0.074)$, respectively, and none of the participants adjusted the fluconazole dose based on body weight. Moreover, $14.3 \%$ would consider fluconazole for the treatment of candidemia caused by C. glabrata, and $8.9 \%$ would use it for C. krusei infections, with no differences between medical specialties. Voriconazole was considered a reasonable secondline option in the treatment of patients with candidemia where fluconazole had failed by $42.9 \%$ of responders, and $75 \%$ of these were ICU physicians $(\mathrm{p}=0.019)$.

Many (33.9\%) physicians would consider fluconazole as the drug of choice in the treatment of unstable patients with candidemia (ID 25\%, ICU 40.6\%; $\mathrm{p}=0.222$ ). Echinocandins were preferred in such scenario by only $33.9 \%$ of the individuals surveyed (ID 45.8\%, ICU $25.0 \%$; $\mathrm{p}=0.103$ ). The recommended caspofungin dosages for patients with candidemia were unknown to a quarter of the physicians interviewed, and $71.4 \%$ of these individuals were ICU physicians. Similarly, anidulafungin dosages were unknown to $46.4 \%$ of the participants, among whom $73 \%$ were ICU physicians.

Most (62.5\%) ICU physicians were not aware of any limitation regarding the use of amphotericin B in C. lusitaniae infections. Additionally, $50 \%$ of ICU specialists were not able to comment on the use of echinocandins for $C$. parapsilosis or $C$. guilliermondii infections ( $<<0.001$ for all comparisons with ID physicians).

For neutropenic patients with candidemia who were not previously exposed to azoles, fluconazole was the drug of choice for $75 \%$ of ICU and ID physicians. In the case of an unstable neutropenic patient, $46.8 \%$ of the ICU physicians preferred a polyene, either deoxycholate amphotericin B (31.2\%) or a 


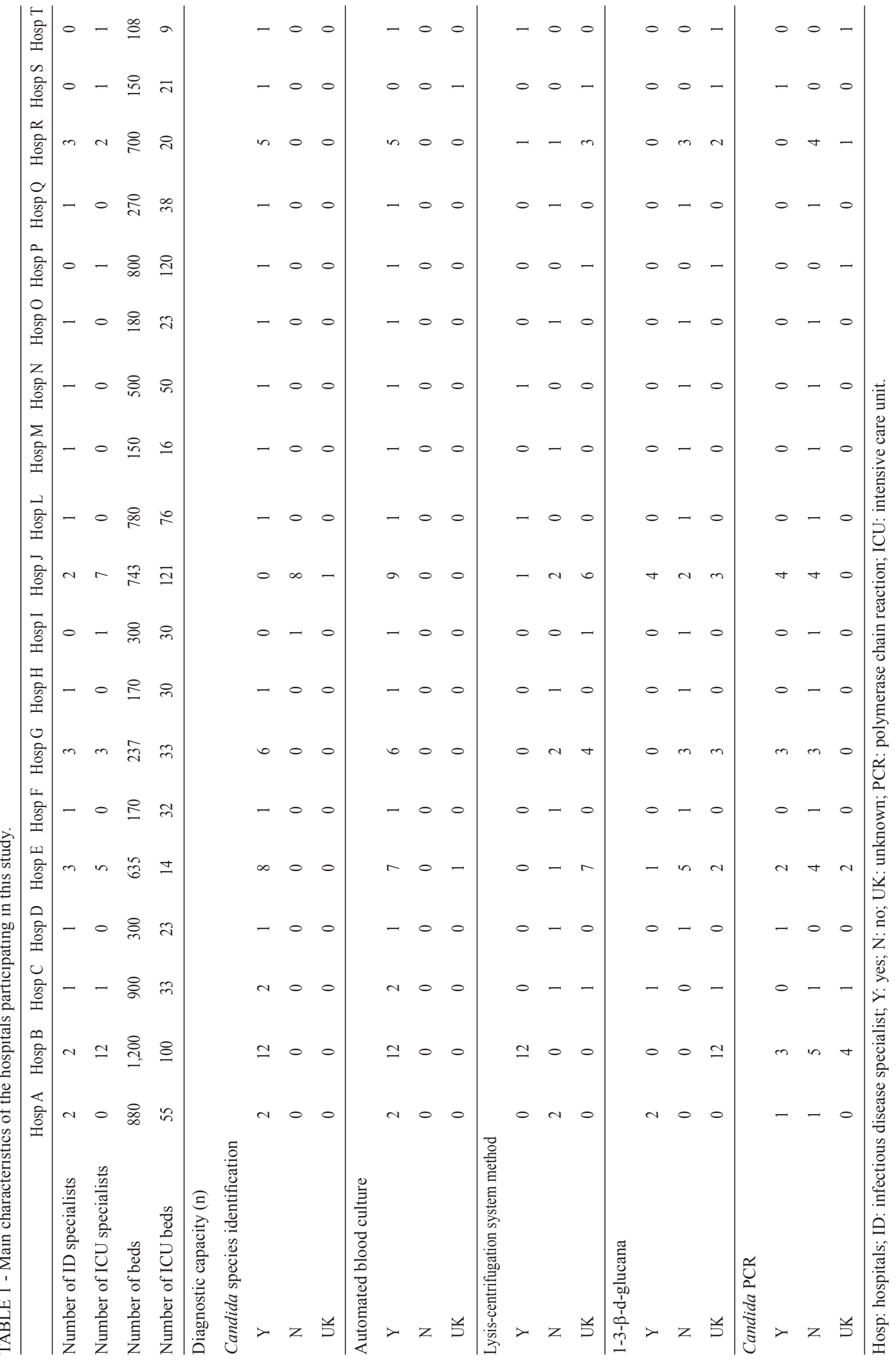


TABLE 2 - The main differences observed between ID and ICU specialists regarding perceptions and management of Candida infections.

\begin{tabular}{|c|c|c|c|}
\hline & $\begin{array}{c}\text { ID } \\
\text { doctors } \\
(\%)\end{array}$ & $\begin{array}{c}\text { ICU } \\
\text { doctors } \\
(\%)\end{array}$ & $\begin{array}{c}\mathrm{P} \\
\text { value }\end{array}$ \\
\hline Perception that candidemia caused by Candida species other than C. albicans occurred in less than $10 \%$ of cases & 31.2 & 69.6 & 0.018 \\
\hline Treatment of all patients with candidemia with antifungal drugs & 95.8 & 65.6 & 0.006 \\
\hline Fluconazole use for neutropenic patients previously exposed to azoles & 12.5 & 46.9 & 0.024 \\
\hline Lack of knowledge on echinocandin dosage & 29.2 & 68.8 & 0.003 \\
\hline Echinocandins as first-choice for unstable neutropenic patients & 50.0 & 18.8 & 0.013 \\
\hline
\end{tabular}

ID: infectious disease specialist; ICU: intensive care unit specialist.

lipid formulation of amphotericin B (15.6\%). Echinocandins were preferred by $50 \%$ and $18.8 \%$ of ID and ICU physicians, respectively, in such a context $(\mathrm{p}=0.013)$.

Combining antifungal drugs for the treatment of invasive Candida infections was an uncommon practice $(10.7 \% ; \mathrm{n}=6)$. Most situations in which such practice was considered were in episodes of Candida endocarditis $(\mathrm{n}=3)$ and meningitis $(\mathrm{n}=2)$, or in the treatment of neutropenic patients $(n=4)$.

\section{Management of central venous catheters}

Systematic removal of short-term management of central venous catheters (CVCs) was the usual practice for $93.8 \%$ of ICU specialists, in contrast to $70.8 \%$ of ID physicians $(\mathrm{p}=0.030$ ). Regarding long-term CVCs, these proportions were $51.6 \%$ and $54.2 \%$, respectively.

\section{Frequency of blood culture after candidemia diagnosis}

Following the diagnosis of candidemia, most participants $(58.9 \%)$ reported obtaining additional blood cultures at $48-72 \mathrm{~h}$ intervals until the clearance of candidemia was documented. No significant differences in this protocol were observed between ID and ICU physicians.

\section{Echocardiographic examination}

The proportion of physicians who requested an echocardiogram for patients with candidemia, persistent fever and blood cultures remaining positive for Candida spp. was $43.8 \%$ and $66.7 \%$ for ICU and ID physicians, respectively $(p=0.089)$. Table1 summarizes the results of the survey.

\section{DISCUSSION}

This study reveals that intensive care and infectious disease physicians in Brazil differ in several ways on how they understand the epidemiology of invasive Candida infections and on the clinical management of such conditions. Most physicians who were selected to participate in the study were based in large tertiary hospitals in which proper diagnostic facilities were apparently in place. Moreover, the epidemiology of candidemia had been well-documented over the preceding years in the medical centers in which these physicians were working ${ }^{1,2,5-7}$. We were surprised to see that, despite enjoying these advantages, a large proportion of the physicians who were interviewed demonstrated little knowledge regarding the epidemiology of invasive Candida infections. For instance, more than half of the participants declared that the frequency of candidemia caused by Candida species other than Candida albicans was lower than $10 \%$; the actual proportion reported in most studies varies from $40 \%$ to $74 \%{ }^{8}$. Additionally, more than $90 \%$ of the physicians were not aware of the increase of C. glabrata incidence that has been documented in some medical centers in Brazil ${ }^{7,14,15}$. As shown in Table 1, misunderstanding of the epidemiology of candidemia was particularly common among the ICU physicians.

In addition, ICU and ID physicians also had different views on the clinical use of antifungal susceptibility testing. Data obtained in this study showed that the ICU physicians were less aware of the importance of identifying Candida at the species level for predicting its susceptibility to antifungal drugs. For instance, C. lusitaniae is known to be amphotericin B-resistant, and both C. guilliermondii and C. parapsilosis have decreased in vitro susceptibility to echinocandins 9 . ICU physicians seemed to be particularly uninformed regarding these associations. In contrast, the frequency of compulsory requests for in vitro susceptibility tests was higher for the ICU physicians than for the ID physicians. This finding could be the result of sampling bias because many of the ICU physicians were working in the same hospital, and the requests for susceptibility tests could reflect institutional protocols. Moreover, many ICU physicians reported a willingness to use voriconazole for candidemic patients in which fluconazole had failed, which demonstrates a lack of 
knowledge regarding cross-resistance in the triazole class. This lack of knowledge may be attributable to ICU physicians being less familiar with the IDSA's guidelines.

Several medical societies, including the IDSA, recognize the importance of antifungal drug treatment for all patients with candidemia ${ }^{3}$. This recommendation is justified by the high mortality associated with candidemia (at least $50 \%$ ) and by the potential for metastatic complications in patients not treated with antifungal drugs ${ }^{2}$. However, $21.4 \%$ of the participants in this study appeared to not systematically treat candidemia, and most of these participants were ICU specialists $(34.4 \%$; $\mathrm{p}=0.008)$. It should be noted that no clinical or laboratory data are available to allow clinicians to safely select patients with transient episodes of candidemia that would not require treatment.

Fluconazole was the first-choice antifungal treatment for most physicians in this study, most likely because of their longstanding familiarity with fluconazole's safety profile and dosing. However, many physicians referenced the use of fixed doses of fluconazole rather than guiding therapy based on body weight; additionally, the administration of loading doses was frequently regarded as unimportant ${ }^{3}$, with ICU and ID physicians registering similar opinions. Fluconazole was also preferred in situations in which the IDSA's guidelines recommend other therapeutic options.

One of the most striking findings of this study was the limited knowledge demonstrated by participants on the echinocandin class of antifungal drugs. Only $45.8 \%$ of ID physicians and $25 \%$ of ICU specialists reported using echinocandins as a first-line treatment for unstable patients with candidemia $(\mathrm{p}=0.154)$. This is in frank contrast to the latest guidelines for treating patients with candidemia ${ }^{3}$; in such a scenario, echinocandins are considered first-choice treatments. The limited availability of this antifungal class in the Brazilian market at the time of the survey in addition to the scarcity of resources at public hospitals, which comprised a large number of our sampling sites, may explain the apparently limited knowledge that the physicians had about this drug.

Removal of central venous catheters has been recommended in the IDSA's guidelines as an adjunct measure in the treatment of patients with candidemia ${ }^{3}$ despite the controversy regarding this subject ${ }^{10}$. In this study, the ICU physicians endorsed a more aggressive approach regarding catheter management: $93.8 \%$ would remove catheters promptly, in comparison to $70.8 \%$ of ID physicians $(\mathrm{p}=0.030)$. As expected, these proportions were reduced to $\sim 50 \%$ when long-term catheters were considered.

Another striking finding of this study was the limited frequency in which eye examination was performed in patients with candidemia, particularly by ICU physicians $(\sim 45 \%)$. Although fungal endophthalmitis is an uncommon condition, it may lead to blindness and requires specific and prolonged treatment once the diagnosis is made. Therefore, eye examination is recommended for all patients with candidemia ${ }^{11}$. In one investigation in Brazil, the frequency of eye examination in patients with candidemia was as low as $7.6 \%{ }^{12}$, which indicates the importance of educating healthcare professionals on this subject.
Consistent with this study's findings, Eggimann et al. ${ }^{13}$ observed that many differences in management approaches between ICU and ID physicians also exist in Switzerland ${ }^{13}$.

The present study reveals the need to better educate physicians in Brazil regarding invasive Candida infection, which is an important and aggressive infection with a high mortality if not correctly identified and treated. Nevertheless, the appropriate management of this disease depends on increasing the drug options available in our country and encouraging global coverage in private and public hospitals, thereby improving health care.

\section{ACKNOWLEDGMENTS}

We wish to thank all physicians who helped make this article possible by responding to the survey.

\section{CONFLICT OF INTEREST}

Drs. Pasqualotto and Colombo have consulted for and received research grants and speaker honoraria from Pfizer, MSD, Astellas and United Medical (Gilead). Dr. Shultz has no conflict of interest to declare.

\section{REFERENCES}

1. Colombo AL, Nucci M, Park BJ, Nouér SA, Arthington-Staggs B, Matta DA, et al. Epidemiology of Candidemia in Brazil: a Nationwide Sentinel Surveillance of Candidemia in Eleven Medical Centers. J Clin Microbiol 2006, 44:2816-2823.

2. Colombo AL, Guimarães T. Epidemiology of hematogenous infections due to Candida spp. Rev Soc Bras Med Trop 2003; 36:599-607.

3. Pappas PG, Kauffman CA, Andes D, Benjamin Jr DK, Calandra TF, Edwards Jr JE, et al. Clinical practice guidelines for the management of candidiasis: 2009 update by the Infectious Diseases Society of America. Clin Infect Dis 2009; 48:503-535.

4. Olaechea PM, Palomar M, León-Gil C, Alvarez-Lerma F, Jordá R, Nolla-Salas $\mathrm{J}$, et al. Economic impact of Candida colonization and Candida infection in the critically ill patient. Eur J Clin Microbiol Infect Dis 2004; 23:323-330.

5. Antunes AG, Pasqualotto AC, Diaz MC, d'Azevedo PA, Severo LC. Candidemia in a Brazilian tertiary care hospital: species distribution and antifungal susceptibility patterns. Rev Inst Med Trop Sao Paulo 2004; 46:239-241.

6. Colombo AL, Guimarães T, Silva LR, Almeida Monfardini LP, Cunha AK, Rady P, et al. Prospective observational study of candidemia in São Paulo, Brazil: incidence rate, epidemiology, and predictors of mortality. Infect Control Hosp Epidemiol 2007; 28:570-576.

7. Pasqualotto AC, Zimerman RA, Alves SH, Aquino VR, Branco D, Wiltgen D, et al. Take control over your fluconazole prescriptions: the growing importance of Candida glabrata as an agent of candidemia in Brazil. Infect Control Hosp Epidemiol 2008; 29:898-899.

8. Nucci M, Queiroz-Telles F, Tobón AM, Restrepo A, Colombo AL. Epidemiology of opportunistic fungal infections in Latin America. Clin Infect Dis 2010; 51:561-570.

9. Lyon GM, Karatela S, Sunay S, Adiri Y. Antifungal susceptibility testing of Candida isolates from the Candida surveillance study. J Clin Microbiol 2010; 48:1270-1275.

10. Pasqualotto AC, Severo LC. The importance of central venous catheter removal in patients with candidaemia: time to rethink our practice? Clin microbial Infect 2008; 14:2-4. 
11. Pérez Blázquez E. Ophthalmoscopic examination in critically ill nonneutropenic patients: Candida endophtalmitis. Rev Iberoam Micol 2006; 23: 16-19.

12. Pasqualotto AC, Severo LC. Why don't we just do what we have to do? $\mathrm{J}$ infect 2005; 51:260-261.

13. Eggimann P, Calandra T, Fluckiger U, Bille J, Glauser MP, Marchetti O. Invasive candidiasis: comparison of management choices by infectious disease and critical care specialists. Intensive Care Med 2005; 31:1514-1521.
14. Colombo AL, Garnica M, Camargo LFA, Cunha CA, Bandeira AC, Borghi D, et al. Candida glabrata: na emerging pathogen in Brazilian tertiary care hospitals. Med Mycol 2013; 51:38-44.

15. Moretti ML, Trabasso P, Lyra L, Fagnani R, Resende MR, Cardoso LGO, et al. Is the incidence of candidemia caused by Candida glabrata increasing in Brazil? Five-year surveillance of Candida bloodstream infection in a university reference hospital in southeast Brazil. Med Mycol 2013; 51: 225-230. 